\title{
BMJ Open Mediating effects of lower extremity function on the relationship between night sleep duration and cardiovascular disease risk: a cross-sectional study in elderly Chinese without cardiovascular diseases
}

Hui Zhang, ${ }^{1,2}$ Xiaoyu Chen, ${ }^{3}$ Peipei Han, ${ }^{2}$ Weibo Ma, ${ }^{1,2}$ Yuanyuan Zhang, ${ }^{3}$ Peiyu Song, ${ }^{3}$ Yunxiao Wu, ${ }^{2}$ Yibo Zhu, ${ }^{2}$ Zhengxing Jiang, ${ }^{2}$ Ming Cai, ${ }^{2}$ Qi Guo, ${ }^{2}$ Hong Wang (i) ${ }^{1,2}$

To cite: Zhang $\mathrm{H}$, Chen $\mathrm{X}$, Han $P$, et al. Mediating effects of lower extremity function on the relationship between night sleep duration and cardiovascular disease risk: a cross-sectional study in elderly Chinese without cardiovascular diseases. BMJ Open 2021;11:e046015. doi:10.1136/ bmjopen-2020-046015

- Prepublication history for this paper is available online. To view these files, please visit the journal online (http://dx.doi. org/10.1136/bmjopen-2020046015).

Received 22 0ctober 2020 Accepted 14 June 2021

Check for updates

(C) Author(s) (or their employer(s)) 2021. Re-use permitted under CC BY-NC. No commercial re-use. See rights and permissions. Published by BMJ.

For numbered affiliations see end of article.

\section{Correspondence to}

Professor Hong Wang; wanghongplus@163.com and

Professor Qi Guo;

guoqijp@gmail.com

\section{ABSTRACT}

Objective The purpose of this study is to investigate the mediating effect of physical performance on the relationship between night sleep duration and risk of cardiovascular disease (CVD) in elderly Chinese without CVD.

Methods 782 participants without CVD over 65 years (average: $70.82 \pm 3.86$ years, 448 women) were included in this study. CVD risk was calculated by the Framingham Risk Score and participants were divided into four groups of night sleep duration (in hours): $<7$ hours, $\geq 7-8$ hours, $>8-9$ hours and $>9$ hours. Upper extremity function was measured by grip strength and lower extremity function was measured by the Short Physical Performance Battery (SPPB), consisting of balance, $4 \mathrm{~m}$ walk and chair stands tests.

Results After adjusting for covariates, long night sleep duration (>9 hours) and CVD risk were significantly positively associated compared with moderate night sleep duration ( $\geq 7-8$ hours) $(\beta=1.152 ; 95 \% \mathrm{Cl} 0.05$ to 2.25 ). SPPB total score (indirect effect $a b=-0.122$ $95 \% \mathrm{Cl}-0.291$ to -0.0008 ) rather than grip strength partially mediated the relationship between long night sleep duration and CVD risk, in which chair stands played a major mediating role (indirect effect $a b=-0.171$; $95 \% \mathrm{Cl}-0.040$ to -0.0006 ), while balance (indirect effect $\mathrm{ab}=-0.016 ; 95 \% \mathrm{Cl}-0.100$ to 0.063 ) and $4 \mathrm{~m}$ walk (indirect effect $\mathrm{ab}=0.048 ; 95 \% \mathrm{Cl}-0.066$ to 0.201 ) did not. Conclusions Lower extremity function, especially lower limb muscle strength, partially mediates a positive association between long night sleep duration and CVD risk in the elderly without CVD. Suitable interventions for physical performance and sleep may minimise the risk of subsequent CVD.

\section{INTRODUCTION}

Cardiovascular disease (CVD) is the leading cause of death worldwide, with an estimated 17 million people dying from CVD in 2017 . $^{1}$
Strengths and limitations of this study

This study is the first to suggest that lower extremity function, especially lower limb muscle strength, partly mediated the association between long night sleep duration and risk of cardiovascular disease (CVD) in elderly Chinese without CVD.

- Physical performance was divided into upper and lower extremity functions, which could more accurately reveal the role of physical performance in night sleep duration and CVD risk and provide new insights into exercise interventions for upper and lower extremity functions in the future.

- Data on night sleep duration were self-reported rather than objectively measured, which could overestimate actual night sleep duration.

- For large population-based studies it is not feasible to objectively assess sleep duration, and selfreported sleep duration is the most frequently used method.

In China, CVD is the disease with the highest disability and mortality among the elderly. The large CVD patient population also comes with a huge burden to the society. ${ }^{2}$ Therefore, prevention of CVD is an important part of Chinese national health policy. Prolonged night sleep duration and decreased physical performance are common in the elderly, and both may affect the occurrence of CVD. Previous studies have shown that healthier physical performance and sleep may mitigate cardiometabolic risk in the elderly, thus helping achieve the goal of preventing $\mathrm{CVD}^{3}{ }^{4}$; however, the relationship between the three and the underlying mechanisms remain unclear. 
Sleep is an important part of daily life and is closely linked to human health conditions. With increased age, about one-third of the elderly have a night sleep duration that is either too long or too short. ${ }^{5}$ Extreme night sleep duration may impair vascular endothelial function by accelerated lipid deposition ${ }^{6}$ and may also cause deterioration of the vasculature by adversely affecting muscle strength. ${ }^{7}$ Although previous studies have suggested that extreme night sleep duration is related to CVD risk, especially in the elderly, the relationship may be J-shaped, ${ }^{89}$ U-shaped ${ }^{10}$ or neither. ${ }^{11}$

Physical performance, including upper and lower extremity functions, declines gradually with age, and poor physical performance in the elderly is strongly associated with adverse health outcomes such as CVD and mortality. ${ }^{12-14}$ Notably, different types of physical performance have different effects on cardiovascular function. Lower extremity function assists the heart in pumping blood, while upper extremity function is closely related to endothelial function. ${ }^{15} 16$ Meanwhile, the relationship between physical performance and night sleep duration has also been previously confirmed. ${ }^{5}$ Thus, physical performance may play a role in the relationship between night sleep duration and CVD risk in the elderly and may even be one of the underlying mechanisms. However, the correlation between night sleep duration and CVD risk remains inconsistent. Few studies have observed that physical performance is simultaneously associated with night sleep duration and CVD risk; the role of physical performance, especially upper and lower extremity functions, is unclear.

The purpose of this study was to explore the relationship between night sleep duration and CVD risk, as well as the mediating effects of upper and lower extremity functions, such as grip strength and the Short Physical Performance Battery (SPPB), on the relationship in elderly Chinese without CVD.

\section{METHODS}

\section{Participants}

The Adult Physical Fitness and Health Cohort Study enrolled 1482 community-dwelling older Chinese adults ( $\geq 65$ years) living in Tianjin and Shanghai from 2018 to 2019, of whom 486 participants with history of CVD (including ischaemic heart disease, stroke, atrial fibrillation and flutter, peripheral artery diseases, etc) were excluded. Participants were invited to provide informed consent and completed the questionnaire, physical measurement, blood pressure assessment and routine blood test. Other exclusion criteria included (1) age $\geq 80$ years; (2) incomplete blood tests and blood pressure assessments; (3) failure to complete specific tests such as grip strength and SPPB for disability or limitation of motion; (4) failure to complete the questionnaire due to severe visual and hearing impairment; (5) diagnosis of obstructive sleep apnea (OSA) and other sleep disorders; and (6) study participation refusal. Following these exclusions, 782 participants (334 men and 448women) were included.

\section{Assessment of CVD risk and collection of biomarker data}

CVD risk was calculated according to the Framingham Risk Score, which is the most widely used CVD risk scoring system globally. Continuous variables are suitable for assessing the 10-year risk of developing CVD in Asian adults, providing convenient and efficient long-term predictions. ${ }^{17}$ The Framingham Risk Score was calculated separately for each sex and takes into account age, total cholesterol, high-density lipoprotein, systolic blood pressure, treatment for hypertension and history of smoking. ${ }^{18}$ Information on age, sex, treatment for hypertension and history of smoking was obtained by trained interviewers through questionnaires. Smokers were defined as any cigarette smoking in the last month.

Participants were required to have fasted overnight (at least 10 hours). An antecubital vein blood sample was drawn and centrifuged for $15 \mathrm{~min}$ at 3000 revolutions per minute (rpm). Fasting plasma glucose, total cholesterol, triglycerides, low-density lipoprotein cholesterol and high-density lipoprotein cholesterol were measured using the Roche Modular P (Roche Diagnostic Company, Switzerland). ${ }^{19}$ Systolic blood pressure and diastolic blood pressure were measured on the upper left limb using the PASESA AVE-2000 (Shisei Datum, Tokyo, Japan) after 10 min of sitting, and blood pressure was measured twice irrespective of the subject's antihypertensive therapy.

\section{Assessment of sleep behaviours}

Information on sleep behaviours was measured by trained interviewers through self-reported questionnaires. Afternoon nap was assessed by asking 'Did you have a habit of afternoon nap in the last month?' Night sleep duration was measured by the question 'In the last month, when did you usually go to bed at night and when did you wake up in the morning?' This interval was used as night sleep duration and was divided into four groups: $<7$ hours (short night sleep duration), $\geq 7-8$ hours, $>8-9$ hours and $>9$ hours (long night sleep duration). According to the National Sleep Foundation, ${ }^{20}$ the optimal night sleep duration is $\geq 7-8$ hours, which was used as the control group.

\section{Performance-based assessment}

Physical performance was measured by objective physical tests including grip strength and the SPPB. Details on grip strength have been previously described. ${ }^{21}$ Briefly, grip strength $(\mathrm{kg})$ was used as a measure of upper extremity strength and was quantified using a handheld dynamometer (GRIP-D; Takei, Niigata, Japan). Participants were asked to use their dominant hand to exert their best effort twice, keeping an upper limb abduction of $45^{\circ}$ and the elbow joint straight throughout the test. Participants made two attempts, with a 1 min interval; if the two results differed more than $10 \%$, participants were told to make a 
third attempt. The average grip strength was used in the analyses.

The SPPB is a validated objective measurement of lower extremity function (balance, $4 \mathrm{~m}$ walk, chair stands). ${ }^{22}$ The battery includes tests for standing balance, a timed 'usual pace' $4 \mathrm{~m}$ walk and repeated chair stands. For the standing balance test, participants were asked to stand with their feet in three positions for $10 \mathrm{~s}$ : feet together, semitandem and full tandem. The walking test required participants to stand with their feet toes touching the starting line and begin walking at their usual pace following verbal commands. ${ }^{21}$ Repeated chair stands needed participants to stand up and sit down from a chair five times as quickly as possible, while keeping their hands folded across their chest, and the completion time was recorded. Each test was scored from 0 to 4 , with higher scores indicating better performance. The three test scores were added, resulting in a summary score of SPPB ranging from 0 to $12 .^{22}$

\section{Assessment of other variables}

Height $(\mathrm{m})$ and weight $(\mathrm{kg})$ were measured using standard protocol. Body mass index (BMI) was calculated as weight/height ${ }^{2}\left(\mathrm{~kg} / \mathrm{m}^{2}\right)$, and BMI $\geq 30 \mathrm{~kg} / \mathrm{m}^{2}$ is defined as obesity. Sociodemographic variables including age, gender, illiteracy, marital status and living status were obtained from a multidimensional questionnaire. Information on smoking and drinking was also obtained from the questionnaire. Subjects who reported a history of more than one fall in the past year were categorised as 'fallers'. ${ }^{23}$ Physical activity was assessed using the short form of the International Physical Activity Questionnaire, the methods of which have been previously described. ${ }^{24}$ Depression symptoms were evaluated by the Geriatric Depression Scale, which consists of 30 items with a total score of $0-30$. Higher scores indicate more severe depressive symptoms. ${ }^{25}$

\section{Statistical analysis}

Data were presented as mean $\pm \mathrm{SD}$ and median (quartiles) for continuous variables and frequency (percentages) for categorical variables. Analysis of variance and Kruskal-Wallis test corrected by Bonferroni were used for continuous variables and $\chi^{2}$ test was used for categorical variables.

All analyses were adjusted for age, sex, BMI, living alone, fallers, afternoon nap and obesity. We used multiple linear regression to identify the association between CVD risk, night sleep duration and physical performance after controlling for all covariables. Mediation analysis was used to investigate the role of mediator variable $(\mathrm{M})$ in the association between independent variable (X) and outcome variable $(\mathrm{Y})$. The mediation model ${ }^{26}$ was performed using the PROCESS macro V.3.5 written by Andrew F Hayes. CVD risk was the dependent variable, physical performance such as grip strength and SPPB was the mediating variable, and night sleep duration was the independent variable. The total effect (path c) represents the sum of the direct and indirect effects of night sleep duration on CVD risk. The direct effect (path c') is the effect of night sleep duration on CVD risk after adjustment for physical performance. Finally, the indirect effect (path ab) is the mediating effect of the association between night sleep duration and CVD risk. In addition, the three parts of SPPB were analysed separately as mediating variables to observe the mediating effects of balance, $4 \mathrm{~m}$ walk and chair stands on night sleep duration and CVD risk. We used the bootstrap approach by the PROCESS macro to confirm the statistical significance of the mediating effect. The indirect effect (mediation effect) was estimated by $95 \%$ CI through 1000 bias-corrected bootstrap samples. As recommended by Hayes, we inferred whether the mediating effects were statistically significant based on $95 \% \mathrm{CI}$, excluding zero. All analyses were performed using IBM's Statistical Package for the Social Sciences (SPSS) V.25. A p value $<0.05$ was considered statistically significant.

\section{Patient and public involvement \\ No patients were involved.}

\section{RESULTS}

A total of 782 participants (334 men and 448 women; average: $70.82 \pm 3.86$ years) were included in the analysis. The characteristics of the participants in our cohort study are shown in table 1 . The number of participants in each group of night sleep duration is as follows: $<7$ hours (short night sleep duration), $\mathrm{n}=67 ; \geq 7-8$ hours, $\mathrm{n}=351$; $>8-9$ hours, $\mathrm{n}=237$; and $>9$ hours (long night sleep duration), $n=127$. Compared with the reference group, participants who slept $>9$ hours were older, had higher Framingham Risk Score, were more likely to live alone, fell more frequently and performed worse in grip strength and chair stands.

Table 2 presents the association between night sleep duration and CVD risk in older adults without CVD using multivariate linear regression analysis. After adjustment for age, sex, BMI, living alone, fallers, afternoon nap and obesity, long night sleep duration ( $>9$ hours) was significantly positively associated with CVD risk ( $\beta=1.152 ; 95 \%$ CI 0.05 to 2.25 ), compared with moderate night sleep duration ( $\geq 7-8$ hours). In the adjusted model, grip strength was not associated with CVD risk ( $\beta=0.024 ; 95 \% \mathrm{CI}-0.03$ to 0.08 ), but SPPB ( $\beta=-0.30 ; 95 \%$ CI -0.53 to -0.07 ), especially walking ( $\beta=-1.24 ; 95 \% \mathrm{CI}-2.16$ to -0.32$)$ and chair stands $(\beta=-0.34 ; 95 \% \mathrm{CI}-0.65$ to -0.03$)$, was negatively correlated with CVD risk.

Regarding the mediation analyses, figure $1 \mathrm{~A}$ and table 3 show the mediating effect of physical performance on the relationship between night sleep duration and CVD risk. Long night sleep duration was also significantly associated with CVD risk through the SPPB total score (direct effect $c^{\prime}=1.274 ; 95 \%$ CI 0.176 to 2.372 ), with the SPPB total score playing a mediating role (indirect effect $\mathrm{ab}=-0.122$; $95 \%$ CI -0.291 to -0.0008$)$. Although long night sleep 
Table 1 Subject characteristics across categories of night sleep duration

\begin{tabular}{|c|c|c|c|c|c|}
\hline \multirow[b]{3}{*}{ Variable } & \multicolumn{4}{|c|}{ Night sleep duration } & \multirow[b]{3}{*}{$P$ value } \\
\hline & \multirow{2}{*}{$\begin{array}{l}<7 \text { hours } \\
(n=67)\end{array}$} & \multirow{2}{*}{$\begin{array}{l}\begin{array}{l}7-8 \text { hours } \\
\text { (reference) }\end{array} \\
(n=351)\end{array}$} & \multirow{2}{*}{$\begin{array}{l}>8-9 \text { hours } \\
(n=237)\end{array}$} & \multirow{2}{*}{$\begin{array}{l}>9 \text { hours } \\
(n=127)\end{array}$} & \\
\hline & & & & & \\
\hline Age, years & $69.68 \pm 3.75$ & $70.46 \pm 3.76$ & $70.89 \pm 3.67$ & $72.29 \pm 4.17^{*}$ & 0.002 \\
\hline Female, n (\%) & $36(53.7)$ & $216(61.5)$ & $129(54.4)$ & $67(52.8)$ & 0.187 \\
\hline BMI, kg/m² & $24.04 \pm 3.22$ & $24.00 \pm 3.51$ & $23.63 \pm 3.41$ & $23.72 \pm 3.35$ & 0.565 \\
\hline Widowed, n (\%) & $16(23.9)$ & 57 (16.2) & 40 (16.9) & 21 (16.5) & 0.496 \\
\hline Living alone, n (\%) & $17(25.4)^{*}$ & $38(10.8)$ & $30(12.7)$ & 17 (13.4) & 0.014 \\
\hline Drinker, n (\%) & 24 (35.8) & $83(23.6)$ & $74(31.2)$ & 38 (29.9) & 0.071 \\
\hline Smoker, n (\%) & $17(25.4)$ & $72(20.5)$ & $42(17.7)$ & $26(20.5)$ & 0.563 \\
\hline SPPB total score & $10.00 \pm 1.29$ & $9.97 \pm 1.70$ & $10.24 \pm 1.65$ & $10.23 \pm 1.86$ & 0.200 \\
\hline Balance score & $3.74 \pm 0.58$ & $3.56 \pm 0.76$ & $3.55 \pm 0.76$ & $3.55 \pm 0.84$ & 0.286 \\
\hline $4 \mathrm{~m}$ walk score & $3.95 \pm 0.20$ & $3.90 \pm 0.40$ & $3.89 \pm 0.39$ & $3.82 \pm 0.53$ & 0.153 \\
\hline Chair stands score & $2.29 \pm 1.14$ & $2.50 \pm 1.25$ & $2.79 \pm 1.16^{\star}$ & $2.85 \pm 1.11^{\star}$ & $<0.001$ \\
\hline Faller, n (\%) & $10(14.9)$ & $46(13.1)$ & $49(20.7)$ & $28(22.0)$ & 0.036 \\
\hline IPAQ, Met/wk & $4746(2439,7728)$ & $4746(2373,9896)$ & $4746(2079,8400)$ & $4200(1736,9733)$ & 0.346 \\
\hline SBP, mm Hg & $131.85 \pm 17.65$ & $131.85 \pm 18.3$ & $131.36 \pm 21.03$ & $131.61 \pm 21.22$ & 0.992 \\
\hline DBP, $\mathrm{mm} \mathrm{Hg}$ & $68.32 \pm 9.43$ & $67.52 \pm 10.37$ & $66.73 \pm 10.94$ & $66.18 \pm 10.74$ & 0.439 \\
\hline $\mathrm{FPG}, \mathrm{mmol} / \mathrm{L}$ & $5.56 \pm 1.09$ & $5.74 \pm 1.39$ & $5.85 \pm 1.56$ & $6.06 \pm 1.83$ & 0.093 \\
\hline
\end{tabular}

Data are mean $\pm S D$, percentage or median (quartile 1, quartile 3).

$P$ value comparison across night sleep duration groups using analysis of variance or Kruskal-Wallis for continuous variables and $\chi^{2}$ test for qualitative variables.

${ }^{*} \mathrm{P}<0.05$, compared with subjects with $7-8$ hours of sleep

BMI, body mass index; DBP, diastolic blood pressure; FPG, fasting plasma glucose; GDS, Geriatric Depression Scale; HDL-C, highdensity lipoprotein cholesterol; IPAQ, International Physical Activity Questionnaire; LDL-C, low-density lipoprotein cholesterol; SBP, systolic blood pressure; SPPB, Short Physical Performance Battery; TCH, total cholesterol; TG, triglycerides.

duration was significantly associated with CVD risk after adjusting for grip strength (direct effect $c^{\prime}=1.228 ; 95 \%$ CI 0.120 to 2.336), grip strength had no mediating effect (indirect effect $\mathrm{ab}=-0.076 ; 95 \% \mathrm{CI}-0.240$ to 0.084 ).

The mediating effects of individual SPPB components were further analysed. Figure $1 \mathrm{~B}$ and table 4 present the mediating effects of balance, $4 \mathrm{~m}$ walk and chair stands on night sleep duration and CVD risk. Although there was a significant correlation between night sleep duration and CVD risk after adjusting for balance (direct effect c'=1.168; $95 \%$ CI 0.069 to 2.267 ), $4 \mathrm{~m}$ walk (direct effect c' $=1.104 ; 95 \%$ CI 0.008 to 2.199 ) and chair stands (direct effect c' $=1.279 ; 95 \%$ CI 0.1752 .382 ), respectively, only chair stands played a mediating role (indirect effect $\mathrm{ab}=-0.171 ; 95 \% \mathrm{CI}-0.040$ to -0.0006 ). Balance (indirect effect $\mathrm{ab}=-0.016 ; 95 \% \mathrm{CI}-0.100$ to 0.063 ) and $4 \mathrm{~m}$ walk (indirect effect $\mathrm{ab}=0.048 ; 95 \% \mathrm{CI}-0.066$ to 0.201 ) did not mediate the relationship between long night sleep duration and CVD risk.

\section{DISCUSSION}

This study indicated that, after adjusting for age, sex, BMI, living alone, fallers, afternoon nap and obesity, long night sleep duration ( $>9$ hours) is positively associated with higher risk of CVD and that SPPB total score plays a mediating role. Further analysis of the role of the SPPB components revealed that only chair stands score partially 
Table 2 Associations of CVD risk with night sleep duration and physical performance in a multiple linear regression analysis

\begin{tabular}{|c|c|c|c|c|}
\hline \multirow[b]{2}{*}{ Variables } & \multicolumn{4}{|c|}{$\beta$ coefficient $(95 \% \mathrm{Cl})$} \\
\hline & Unadjusted model & $P$ value & Adjusted model & $P$ value \\
\hline \multicolumn{5}{|c|}{ Night sleep duration } \\
\hline 7-8hours & Ref & Ref & Ref & Ref \\
\hline$>8-9$ hours & $0.69(-0.52$ to 1.90$)$ & 0.260 & $-0.04(-0.92$ to 0.84$)$ & 0.928 \\
\hline SPPB & $-0.31(-0.54$ to -0.08$)$ & 0.008 & $-0.30(-0.53$ to -0.07$)$ & 0.009 \\
\hline Balance & $-0.31(-0.82$ to 0.20$)$ & 0.233 & $-0.31(-0.82$ to 0.19$)$ & 0.223 \\
\hline Walking & $-1.17(-2.09$ to -0.25$)$ & 0.013 & $-1.24(-2.16$ to -0.32$)$ & 0.008 \\
\hline Chair stands & $-0.33(-0.64$ to -0.02$)$ & 0.037 & $-0.34(-0.65$ to -0.03$)$ & 0.033 \\
\hline
\end{tabular}

Significant effects are denoted in bold.

Adjusted model: adjusted for age, sex, body mass index, living alone, faller, afternoon nap and obesity.

CVD, cardiovascular disease; Ref, reference; SPPB, Short Physical Performance Battery.

mediates the association between night sleep duration and CVD risk, while balance and $4 \mathrm{~m}$ walk have no mediating effect. To our knowledge, this is the first study to explore the mediating effects of physical performance on the association between night sleep duration and CVD risk in elderly community-dwelling Chinese.

A
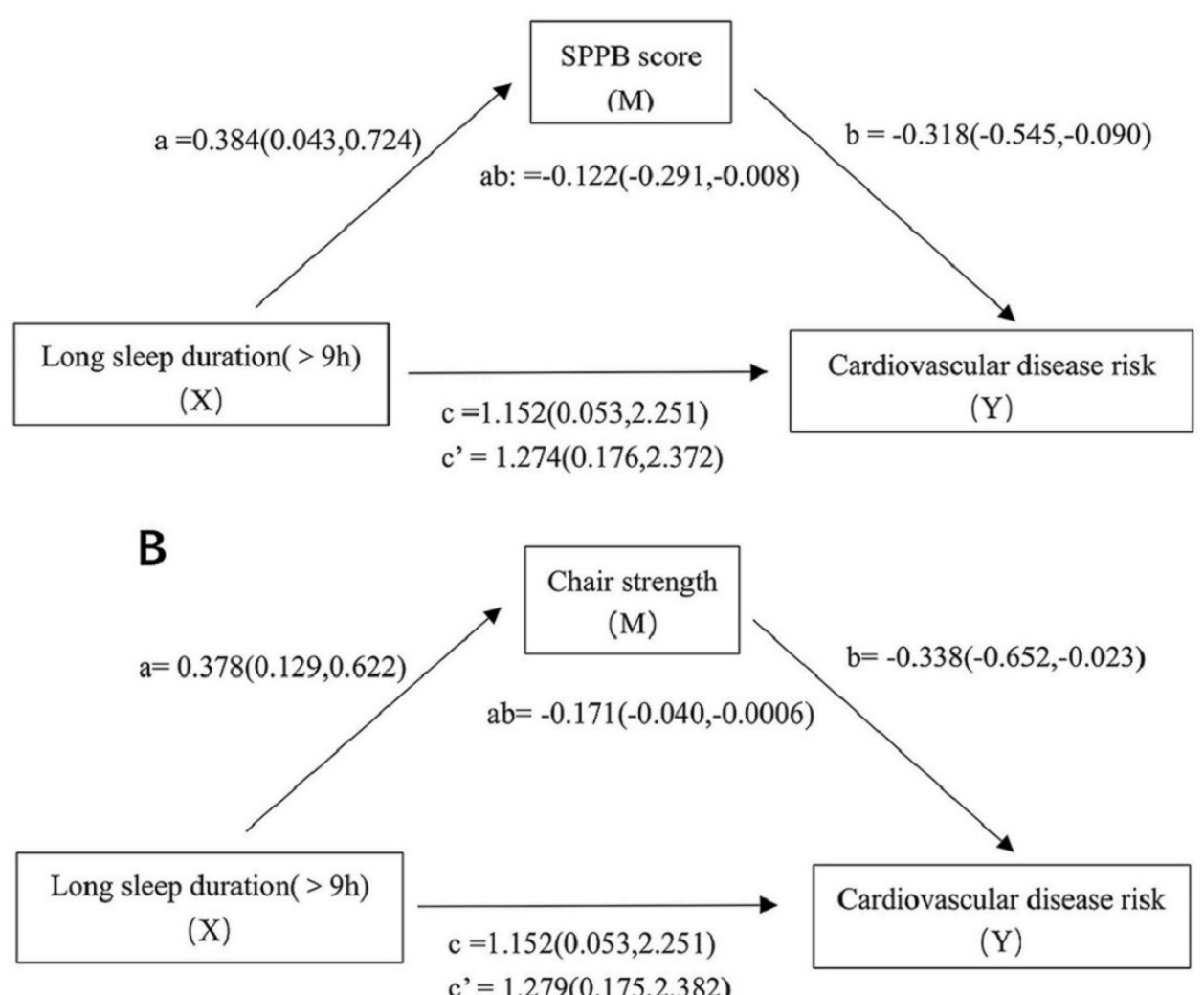

Figure 1 Mediation model (A) for the relationship between long sleep duration and CVD risk, mediated by SPPB score; and (B) for the relationship between long sleep duration and CVD risk, mediated by chair stands. X: independent variable; Y: dependent variable; M: mediator variable. Paths a, b, c and c' are presented as unstandardised coefficients $(95 \% \mathrm{Cl})$. Lower limit $\mathrm{Cl}$, upper limit $\mathrm{Cl}$ : lower and upper levels for $95 \%$ bias-corrected $\mathrm{Cls}$ of the indirect effects based on $1000 \mathrm{bootstraps.} \mathrm{a}=\mathrm{X}$ on $\mathrm{M}$; $\mathrm{b}=\mathrm{M}$ on $Y$; c=total effect of $X$ on $Y$; $c^{\prime}=$ direct effect of $X$ on $Y$; ab=indirect effect of $X$ on $Y$. Covariates: age, sex, body mass index, living alone, faller, afternoon nap and obesity. CVD, cardiovascular disease; SPPB, Short Physical Performance Battery. 


\begin{tabular}{|c|c|c|c|c|c|}
\hline \multirow{2}{*}{$\begin{array}{l}\begin{array}{l}\text { Independent } \\
\text { variable }\end{array} \\
\text { Night sleep } \\
\text { duration }\end{array}$} & \multirow{2}{*}{$\begin{array}{l}\begin{array}{l}\text { Mediating } \\
\text { variable }\end{array} \\
\text { Physical } \\
\text { performance }\end{array}$} & \multirow{2}{*}{$\begin{array}{l}\begin{array}{l}\text { Dependent } \\
\text { variable }\end{array} \\
\text { CVD risk }\end{array}$} & \multicolumn{3}{|c|}{ Coefficient (bias-corrected bootstrap $95 \% \mathrm{Cl}$ ) } \\
\hline & & & Indirect effect (ab) & Total effect (c) & Direct effect (c') \\
\hline$<7$ hours & SPPB & & $-0.0003(-0.151$ to 0.133$)$ & $0.184(-1.222$ to 1.590$)$ & $0.184(-1.216$ to 1.585$)$ \\
\hline 7-8hours & & & Ref & Ref & Ref \\
\hline$>8-9$ hours & & & $-0.090(-0.237$ to 0.0003$)$ & $-0.405(-0.923$ to 0.842$)$ & $0.050(-0.832$ to 0.931$)$ \\
\hline \multirow[t]{2}{*}{$>9$ hours } & & & $-0.122(-0.291$ to -0.008$)$ & $1.152(0.053$ to 2.251$)$ & 1.274 (0.176 to 2.372$)$ \\
\hline & Grip strength & & & & \\
\hline$<7$ hours & & & $0.039(-0.052$ to 0.169$)$ & $0.184(-1.222$ to 1.590$)$ & $0.145(-1.263$ to 1.553$)$ \\
\hline 7-8hours & & & Ref & Ref & Ref \\
\hline$>8-9$ hours & & & $-0.022(-0.101$ to 0.031$)$ & $-0.405(-0.923$ to 0.842$)$ & $-0.019(-0.902$ to 0.865$)$ \\
\hline$>9$ hours & & & $-0.076(-0.240$ to 0.084$)$ & $1.152(0.053$ to 2.251$)$ & $1.228(0.120$ to 2.336$)$ \\
\hline
\end{tabular}

$\mathrm{n}=782$.

Significant effects are denoted in bold.

Covariates: age, sex, body mass index, living alone, faller, afternoon nap and obesity.

CVD, cardiovascular disease; Ref, reference; SPPB, Short Physical Performance Battery.

\section{Night sleep duration and CVD risk}

According to recommendations from the National Sleep Foundation, ${ }^{20}$ the optimal night sleep duration for older adults is $7-8$ hours. Current evidence suggests adults in this group have the lowest risk of CVD..$^{10}$ A cross-sectional study in Japan showed an association between sleep $\leq 4$ hours and coronary heart disease ${ }^{9}$; however, another study showed a weaker link between CVD and reduction of night sleep duration than increment of night sleep duration. ${ }^{10}$ This inconsistent relationship may be partly due to the unclear definition of long sleep and short sleep duration. However, another study showed that the link between decreased sleep time and CVD was weaker than the link between increased sleep time and CVD.

Compared with a night sleep duration of 7-8hours, long night sleep duration ( $>9$ hours) was associated with CVD risk, while short night sleep duration ( $<7$ hours) was not. Similar to the J-shaped association found here, a 21-country longitudinal study found that long night sleep duration was significantly associated with CVD during follow-up, ${ }^{8}$ and this association was more pronounced in older subjects. The relationship between long night sleep duration and CVD risk has not been reflected in other previous studies, possibly due to the lower average age of the population compared with this study $(48.63 \pm 9.57$ years vs $70.82 \pm 3.86$ years) ${ }^{27}$ Older adults are more likely to have comorbidities, which could lead to prolonged sleep. ${ }^{28}$ This may reflect long-standing disease processes; even if these diseases may not yet be clinically apparent, they will increase the risk of CVD.

Abnormal lipid profile is also important. Higher triglycerides and cholesterol related to lipid deposition appear in long sleepers. ${ }^{6}$ Lipid deposition in the large and middle arteries promotes the formation of atherosclerotic plaques, thickening and hardening of arterial walls, narrowing of the lumen, and ultimately leading to ischaemic diseases. In the Fasa Cohort Study, short night sleep duration was associated with a higher predicted risk of $\mathrm{CVD},{ }^{27}$ possibly attributable to lower cholesterol and triglyceride levels compared with the subjects in this study. We hypothesise that lipid profile was also an important factor in the relationship between long night sleep duration and CVD risk. Taking into account the intimate connection between better physical performance and lower blood profile in previous studies, ${ }^{29}$ we included physical performance in the study of night sleep duration and CVD risk.

\section{Physical performance, night sleep duration and CVD risk}

A partial mediating effect of SPPB total score on the association between long night sleep duration and CVD risk was observed, which agrees with previous studies. The InCHIANTI study indicated that long night sleep duration could predict a greater decline in SPPB total score than moderate or short night sleep duration, ${ }^{30}$ and we have previously reported an association between long night sleep duration and poorer physical performance. ${ }^{5}$ Indeed, loss of muscle strength is an important cause of reduced physical performance, and the relationship between upper or lower extremity muscle strength and long night sleep duration has also been widely reported. ${ }^{31} 32$

Moreover, there is evidence that physical performance per se may also be used as a predictor of CVD. Cohort studies have observed a significant inverse linear doseresponse relationship between SPPB total score and CVD. ${ }^{12}$ UK Biobank research suggests that adding grip strength to existing risk scores improved the prediction of CVD. ${ }^{13}$

However, our study indicated that SPPB total score rather than grip strength was associated with CVD risk. These findings are in agreement with the study by Gubelmann et alfrom Switzerland. ${ }^{33}$ While both of these studies used the Framingham Risk Score, the UK Biobank 


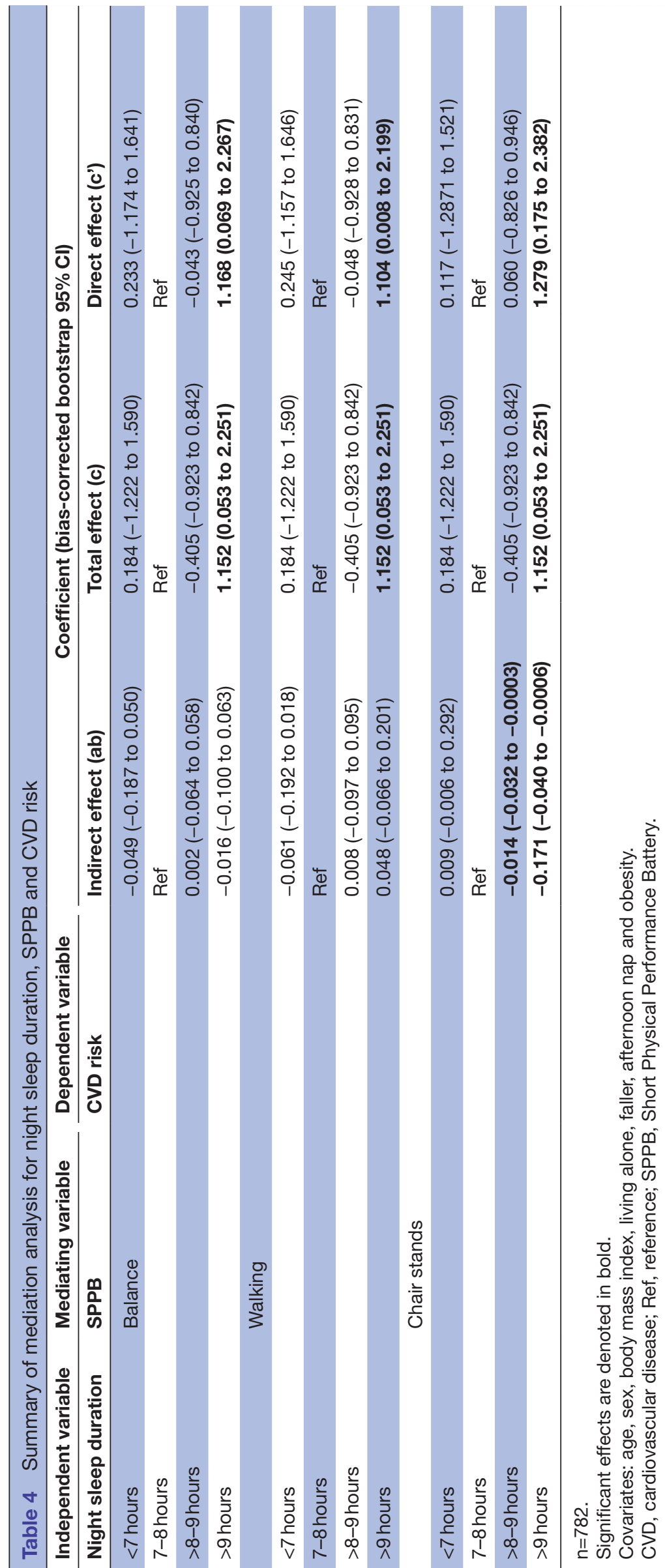

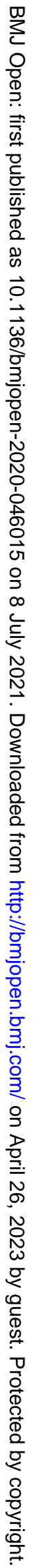


study used the American College of Cardiology/American Heart Association (ACC/AHA) risk score for CVD risk prediction; this difference in formula may account for the difference in the results. ACC/AHA risk score assesses a higher risk of CVD than the Framingham Risk Score, with the ACC/AHA guidelines recommending that the risk threshold for drug treatment is $7.5 \%$ while the high-risk threshold for the Framingham Risk Score is $20 \%{ }^{18}{ }^{34}$ A population study suggested that ACC/AHA risk scores seem to be too sensitive, and its assessment of cardiovascular risk is significantly higher than the Framingham Risk Score and other cardiovascular risk equations. ${ }^{35}$ These differences may explain the absence of a correlation between grip strength and CVD risk in this study.

The different effects of upper and lower extremity functions on the cardiovascular system may be an important factor. On the one hand, upper extremity function is closely related to endothelial function, ${ }^{16}$ and a study indicated a significant correlation between low grip strength and arterial stiffness, while another did not. ${ }^{16}{ }^{36}$ Lower extremity muscle could assist the heart in pumping blood ${ }^{15}$ and SPPB score has been shown to predict CVD risk. ${ }^{37}$ Because lower extremity muscles such as quadriceps femoris and triceps surae are larger and stronger upper limb, they have greater impact on the compression and relaxation of blood vessels and the return of venous blood to the right atrium. ${ }^{15}$ On the other hand, chronic inflammation, as an important mechanism of CVD, indirectly reduces physical performance through increased skeletal muscle proteolysis, ${ }^{38}$ and the muscle strength of the lower extremity is decreased more rapidly with age than that of the upper extremity. ${ }^{39}$ Studies have found that chronic inflammation, especially high C-reactive protein (CRP) levels, is more significantly associated with lower extremity muscle strength. ${ }^{40}$ As a consequence, we hypothesise that lower extremity function may be a potential mechanism in the relationship between long night sleep duration and CVD risk.

Further analysis examined the role of the SPPB components, and only chair stands played a mediating role in night sleep duration and CVD risk. Chair stands represent the strength of the lower limb, which is not only related to mobility function, but also closely to vascular risk burden such as hypertension, type 2 diabetes and obesity. ${ }^{71}$ SPPB is commonly used to evaluate the prognosis of patients with CVD, and lower limb muscle strength can even predict the risk of CVD mortality independent of physical activity. ${ }^{37}$ Thigh muscle attenuation is more prevalent in people with longer night sleep duration, and lower extremity function and long night sleep duration is also correlated. ${ }^{54}$ Considering that improvement of physical performance and sleep can significantly reduce the risk of cardiometabolic, lower limb muscle strength played a mediating role in the relationship between long night sleep duration and CVD risk, and targeted interventions for exercise and sleep may prevent CVD.

\section{Strengths and limitations}

This study is the first to suggest that lower extremity function, especially lower limb muscle strength, partially mediated the relationship between long night sleep duration and CVD risk. This may provide a reference for clinical medicine for preventing CVD. As a simple and easily calculated CVD risk prediction model, the Framingham Risk Score is widely used to effectively assess the CVD risk of a population without CVD within 10 years. This aids in the prevention and control of CVD risk factors targeted at high-risk individuals. Another strength of this study is that physical performance was divided into upper and lower extremity functions. This could more accurately reveal the role of physical performance in night sleep duration and CVD risk and provide new insights into exercise interventions for upper and lower extremity functions in the future.

This study also has some limitations. First, information about night sleep duration was obtained from selfreported questionnaires rather than objectively assessed. Night sleep duration recorded by actigraphy-measured assessments is more accurate, but it is not feasible to obtain objective sleep data in large population studies. Self-reported night sleep duration has been the most commonly used method and is moderately consistent with actigraphy-measured assessments. ${ }^{43}$ Second, the specific nap time has not been recorded and studies have reported a relationship between daytime naps and CVD in adults. ${ }^{8}$ Therefore, we included afternoon nap as a covariate; however, it did not change our conclusion. Third, we excluded people who had previously been diagnosed with OSA and other sleep disorders; however, the diagnosis of OSA was based on polysomnography, which was difficult to perform in large population studies. We included obesity (BMI $\left.\geq 30 \mathrm{~kg} / \mathrm{m}^{2}\right)$ and men as substitutes for OSA in the analysis of covariates, but the conclusion did not change. Fourth, our study is a cross-sectional study and could not prove causality; however, mediation analysis could further analyse potential mechanisms based on association. ${ }^{26}$ Furthermore, the Framingham Risk Score is a predictive model, and the relationship between night sleep duration and physical performance and the incidence of CVD needs to be further confirmed in longitudinal studies. Finally, the number of participants was limited and the results of this study may not be extended to people of other race, region and age group.

\section{CONCLUSIONS}

Our study shows that, compared with moderate night sleep duration, there is a significant association between long night sleep duration and CVD risk in elderly Chinese without CVD. Lower extremity function, especially lower limb muscle strength, had a mediating effect on the relationship between long night sleep duration and CVD risk. This suggests that physical performance is the underlying mechanism of night sleep duration and CVD risk, and the prevention of CVD may be aided by suitable exercise and 
sleep interventions, especially testing interventions, to minimise the risk of future CVD.

\section{Author affiliations}

${ }^{1}$ Shanghai University of Traditional Chinese Medicine, Shanghai, China

${ }^{2}$ College of Rehabilitation Sciences, Shanghai University of Medicine and Health

Sciences, Shanghai, China

${ }^{3}$ Department of Rehabilitation Medicine, Tianjin Medical University, Tianjin, China

Acknowledgements The authors thank Guiyan Shao from the Chadian public health centre, Xiaofang Ren from Hangu welfare house and Xiaoyue Gu from the Chongming public health centre for providing place and organisation.

Contributors $\mathrm{HZ}$ and $\mathrm{XC}$ completed the statistical analyses and drafted the manuscript. WM, YZ, PS, YW, YC and ZJ contributed to data collection, data entry and data cleaning. $\mathrm{PH}$ and $\mathrm{MC}$ contributed to data analysis and interpretation of study results. QG and HW contributed to acquisition of funding and study design and provided administrative support. All authors wrote the manuscript and approved the final version.

Funding This work was supported by a grant (20YF1418200) from the Shanghai Sailing Programme and a grant (20YJCZH001) from the Youth Fund Project of Research Planning Foundation on Humanities and Social Sciences of the Ministry of Education. The funders had an important role in study design, data collection and analysis, and decision to publish.

Competing interests None declared.

Patient and public involvement Patients and/or the public were not involved in the design, or conduct, or reporting, or dissemination plans of this research. Refer to the Methods section for further details.

Patient consent for publication Obtained.

Ethics approval The study was approved by the ethics committee of Tianjin Medical University and Shanghai University of Medicine and Health Sciences. Research was carried out according to the principles of the Declaration of Helsinki. All records were anonymised and no individual information is identifiable.

Provenance and peer review Not commissioned; externally peer reviewed.

Data availability statement All data relevant to the study are included in the article or uploaded as supplementary information.

Open access This is an open access article distributed in accordance with the Creative Commons Attribution Non Commercial (CC BY-NC 4.0) license, which permits others to distribute, remix, adapt, build upon this work non-commercially, and license their derivative works on different terms, provided the original work is properly cited, appropriate credit is given, any changes made indicated, and the use is non-commercial. See: http://creativecommons.org/licenses/by-nc/4.0/.

ORCID iD

Hong Wang http://orcid.org/0000-0002-2169-051X

\section{REFERENCES}

1 GBD 2017 Causes of Death Collaborators. Global, regional, and national age-sex-specific mortality for 282 causes of death in 195 countries and territories, 1980-2017: a systematic analysis for the global burden of disease study 2017. Lancet 2018;392:1736-88.

2 Liu S, Li Y, Zeng X, et al. Burden of cardiovascular diseases in China, 1990-2016: findings from the 2016 global burden of disease study. JAMA Cardiol 2019;4:342-52.

3 Hoevenaar-Blom MP, Spijkerman AMW, Kromhout D, et al. Sufficient sleep duration contributes to lower cardiovascular disease risk in addition to four traditional lifestyle factors: the MORGEN study. Eur $J$ Prev Cardiol 2014;21:1367-75.

4 Shiroma EJ, Cook NR, Manson JE, et al. Strength training and the risk of type 2 diabetes and cardiovascular disease. Med Sci Sports Exerc 2017;49:40-6.

5 Fu L, Jia L, Zhang W, et al. The association between sleep duration and physical performance in Chinese community-dwelling elderly. PLoS One 2017;12:e0174832.

6 Full KM, Gallo LC, Malhotra A, et al. Modeling the cardiometabolic benefits of sleep in older women: exploring the 24-hour day. Sleep 2020;43. doi:10.1093/sleep/zsz205. [Epub ahead of print: 13 Jan 2020].
7 Jor'dan AJ, Jacob ME, Leritz E, et al. Neuromuscular attributes are associated with poor mobility in older adults with vascular risk conditions. J Frailty Aging 2020;9:30-6.

8 Wang C, Bangdiwala SI, Rangarajan S, et al. Association of estimated sleep duration and naps with mortality and cardiovascular events: a study of 116632 people from 21 countries. Eur Heart $J$ 2019;40:1620-9.

9 Ikehara S, Iso H, Date C, et al. Association of sleep duration with mortality from cardiovascular disease and other causes for Japanese men and women: the JACC study. Sleep 2009;32:295-301.

10 Yin J, Jin X, Shan Z, et al. Relationship of sleep duration with allcause mortality and cardiovascular events: a systematic review and dose-response meta-analysis of prospective cohort studies. J Am Heart Assoc 2017;6. doi:10.1161/JAHA.117.005947. [Epub ahead of print: 09 Sep 2017].

11 Li F, Sun K, Lin D, et al. Longtime napping is associated with cardiovascular risk estimation according to Framingham risk score in postmenopausal women. Menopause 2016;23:950-6.

12 Bellettiere J, Lamonte MJ, Unkart J, et al. Short physical performance battery and incident cardiovascular events among older women. J Am Heart Assoc 2020;9:e016845.

13 Welsh CE, Celis-Morales CA, Ho FK, et al. Grip strength and walking PACE and cardiovascular disease risk prediction in 406,834 UK Biobank participants. Mayo Clin Proc 2020;95:879-88.

14 Strand $\mathrm{BH}$, Cooper R, Bergland $\mathrm{A}$, et al. The association of grip strength from midlife onwards with all-cause and cause-specific mortality over 17 years of follow-up in the Troms $\varnothing$ Study. J Epidemiol Community Health 2016;70:1214-21.

15 Halkar M, Medina Inojosa J, Liedl D, et al. Calf muscle pump function as a predictor of all-cause mortality. Vasc Med 2020;25:20953212.

16 Yoo J-I, Kim M-J, Na J-B, et al. Relationship between endothelial function and skeletal muscle strength in community dwelling elderly women. J Cachexia Sarcopenia Muscle 2018;9:1034-41.

17 Selvarajah S, Kaur G, Haniff J, et al. Comparison of the Framingham risk score, score and $\mathrm{WHO} / \mathrm{ISH}$ cardiovascular risk prediction models in an Asian population. Int J Cardiol 2014;176:211-8.

18 Expert Panel on Detection, Evaluation, and Treatment of High Blood Cholesterol in Adults. Executive summary of the third report of the National cholesterol education program (NCEP) expert panel on detection, evaluation, and treatment of high blood cholesterol in adults (adult treatment panel III). JAMA 2001;285:2486-97. doi:10.1001/jama.285.19.2486

19 Han P, Yu H, Ma Y, et al. The increased risk of sarcopenia in patients with cardiovascular risk factors in Suburb-Dwelling older Chinese using the AWGS definition. Sci Rep 2017;7:9592.

20 Hirshkowitz M, Whiton K, Albert SM, et al. National sleep Foundation's updated sleep duration recommendations: final report. Sleep Health 2015;1:233-43.

21 Shen S, Li J, Guo Q, et al. Body mass index is associated with physical performance in suburb-dwelling older Chinese: a crosssectional study. PLoS One 2015;10:e0119914.

22 Fukumori N, Yamamoto Y, Takegami M, et al. Association between hand-grip strength and depressive symptoms: locomotive syndrome and health outcomes in Aizu cohort study (LOHAS). Age Ageing 2015;44:592-8.

23 Fu L, Yu X, Zhang W, et al. The relationship between sleep duration, falls, and muscle mass: a cohort study in an elderly Chinese population. Rejuvenation Res 2019;22:390-8.

24 Zhang W, Shen S, Wang W, et al. Poor lower extremity function was associated with pre-diabetes and diabetes in older Chinese people. PLoS One 2014;9:e115883.

25 Yang $\mathrm{B}, \mathrm{Yu} \mathrm{H}$, Xing $\mathrm{M}$, et al. The relationship between cognition and depressive symptoms, and factors modifying this association, in Alzheimer's disease: a multivariate multilevel model. Arch Gerontol Geriatr 2017;72:25-31.

26 Hayes AF, Preacher KJ. Statistical mediation analysis with a multicategorical independent variable. Br J Math Stat Psychol 2014;67:451-70.

27 Yazdanpanah MH, Homayounfar R, Khademi A, et al. Short sleep is associated with higher prevalence and increased predicted risk of cardiovascular diseases in an Iranian population: Fasa Persian cohort study. Sci Rep 2020;10:4608.

28 Smagula SF, Koh W-P, Wang R, et al. Chronic disease and lifestyle factors associated with change in sleep duration among older adults in the Singapore Chinese Health study. J Sleep Res 2016;25:57-61.

29 Lawman HG, Troiano RP, Perna FM, et al. Associations of relative handgrip strength and cardiovascular disease biomarkers in U.S. adults, 2011-2012. Am J Prev Med 2016;50:677-83.

30 Stenholm S, Kronholm E, Bandinelli S, et al. Self-reported sleep duration and time in bed as predictors of physical function decline: results from the InCHIANTI study. Sleep 2011;34:1583-93. 
31 Kim M, Yoshida H, Sasai H, et al. Association between objectively measured sleep quality and physical function among communitydwelling oldest old Japanese: a cross-sectional study. Geriatr Gerontol Int 2015;15:1040-8.

32 Lee P-F, Ho C-C, Yeh D-P, et al. Cross-sectional associations of physical fitness performance level and sleep duration among older adults: results from the National physical fitness survey in Taiwan. Int $J$ Environ Res Public Health 2020;17. doi:10.3390/ijerph17020388. [Epub ahead of print: 07 Jan 2020].

33 Gubelmann C, Vollenweider P, Marques-Vidal P. No association between grip strength and cardiovascular risk: the CoLaus population-based study. Int J Cardiol 2017;236:478-82.

34 Goff DC, Lloyd-Jones DM, Bennett G, et al. 2013 ACC/AHA guideline on the assessment of cardiovascular risk: a report of the American College of Cardiology/American heart association Task force on practice guidelines. Circulation 2014;129:S49-73.

35 Motamed N, Rabiee B, Perumal D, et al. Comparison of cardiovascular risk assessment tools and their guidelines in evaluation of 10-year CVD risk and preventive recommendations: a population based study. Int J Cardiol 2017;228:52-7.

36 van Dijk SC, Swart KMA, Ham AC, et al. Physical fitness, activity and Hand-Grip strength are not associated with arterial stiffness in older individuals. J Nutr Health Aging 2015;19:779-84.

37 Campo G, Maietti E, Tonet E, et al. The assessment of scales of frailty and physical performance improves prediction of major adverse cardiac events in older adults with acute coronary syndrome. J Gerontol A Biol Sci Med Sci 2020;75:1113-9.

38 Ferrucci L, Penninx BWJH, Volpato S, et al. Change in muscle strength explains accelerated decline of physical function in older women with high interleukin-6 serum levels. J Am Geriatr Soc 2002;50:1947-54.

39 Ditroilo M, Forte R, Benelli $\mathrm{P}$, et al. Effects of age and limb dominance on upper and lower limb muscle function in healthy males and females aged 40-80 years. J Sports Sci 2010;28:667-77.

40 Sousa ACPA, Zunzunegui M-V, Li A, et al. Association between C-reactive protein and physical performance in older populations: results from the International mobility in aging study (IMIAS). Age Ageing 2016;45:274-80.

41 Sanderson WC, Scherbov S, Weber D, et al. Combined measures of upper and lower body strength and subgroup differences in subsequent survival among the older population of England. J Aging Health 2016;28:1178-93.

42 Marques EA, Figueiredo P, Gudnason V, et al. Associations of 24hour sleep duration and CT-derived measurements of muscle and bone: the AGES-Reykjavik study. Exp Gerontol 2017;93:1-6.

43 Jackson CL, Patel SR, Jackson WB, et al. Agreement between self-reported and objectively measured sleep duration among white, black, Hispanic, and Chinese adults in the United States: multi-ethnic study of atherosclerosis. Sleep 2018;41. doi:10.1093/sleep/zsy057. [Epub ahead of print: 0106 2018] 